\title{
Measuring Sound Absorption: Considerations on the Measurement of the Active Acoustic Power
}

\author{
E. R. Kuipers, Y. H. Wijnant, A. de Boer \\ Research Chair of Structural Dynamics and Acoustics, Faculty of Engineering Technology, \\ University of Twente, P.O. Box 217, 7500 AE, Enschede, The Netherlands. \\ [e.r.kuipers, y.h.wijnant, a.deboer]@ctw.utwente.nl
}

\begin{abstract}
Summary
Using a local plane wave assumption, one can determine the normal incidence sound absorption coefficient of a surface by measuring the acoustic pressure and the particle velocity normal to that surface. As the measurement surface lies in front of the material surface, the measured active and incident acoustic power will generally deviate from those at the material surface, leading to a possibly inaccurate sound absorption coefficient. This phenomenon is particularly pronounced for poorly absorbing surfaces if sound is not normally incident over the whole material surface. Based on an analytical model, it is shown that the accuracy can be improved by extending the measurement surface upon which the active acoustic power is measured. Experimental results demonstrate the usefulness of this approach, in particular for poorly absorbing surfaces.
\end{abstract}

PACS no. 43.20.El, 43.55.Ev, 43.55.Dt

\section{Introduction}

For the measurement of the sound absorption coefficient a large number of methods is available. These methods have in common that all of them rely on an overall sound field model, i.e. a model that describes the whole sound field in front of the surface under investigation. Examples are the diffuse sound field model in the reverberation room method according to ISO 354 [1] and the plane wave model in the tube-based methods according to parts 1 and 2 of ISO 10534 [2, 3]. Whereas these methods are based on an overall plane wave assumption and a directional pattern, methods for measurement in a semi-free field generally rely on a typical sound field model that is more restrictive.

This sound field model consists of a semi-free field that is bounded by a planar, locally-reacting, surface, as for instance described by Attenborough [4]. It will be referred to as the reference model hereafter. A number of methods [5, 6, 7, 8] assume plane wave incidence, whereas other methods consider spherical wave incidence $[9,10,11,12,13,14,15,16]$. A different approach is applied in the in situ method by Takahashi et al. [7], further studied by Otsuru [17], and Din [8]. Although this method yields good results for a large variety of sound fields, it also relies on an overall model, comprising a planar, locally reacting surface subjected to diffuse incidence of plane waves.

Received 7 March 2013

accepted 24 September 2013.
The use of an reference model that describes the overall sound field is likely to lead to inaccuracies if the actual sound field deviates from the model. The first cause for such a deviation may be the presence of reflections other than from the specimen, such as room reflections. A strategy to eliminate these, is to gate these so-called parasitic or spurious reflections out from the impulse response by employing a time-windowing technique $[9,10,11,12,16]$. For methods that do not use an impulse response, one may place the source near the surface [18] so that the relative amplitudes of the unwanted reflections are reduced.

Although such strategies can also be used to gate out, or reduce the effect of, edge-diffracted waves, the use of an overall sound field model may still lead to inaccuracies, even if the measurement is performed in a semianechoic room. Possible causes are multiple, for instance non-omnidirectionally radiating sound sources, samples that are non-locally reacting, or samples having a nonplanar surface. Fitting the reference model parameters to the measurement results will then typically lead to sound absorption coefficients that are inaccurate to a greater or lesser extent $[14,19]$.

Therefore, we developed a measurement method that can deal with non-ideal sound fields. This novel measurement method employs a Local Plane Wave Assumption, and is therefore called the LPW-method. It yields the exact sound absorption coefficient for plane waves that are normally incident upon a locally-reacting surface, but also yields accurate results for near-normal incidence, even if additional reflections are present [20, 21, 22, 23]. By using the LPW-method, at a small distance from the surface of interest, one can straightforwardly determine the local, 


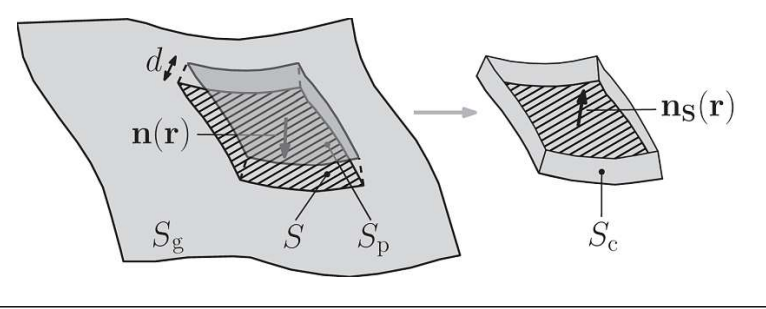

Figure 1. A general, arbitrarily shaped, surface $S_{\mathrm{g}}$, with the surface of interest $S$ (hatched) and $S_{\mathrm{p}}$, being the projection of $S$ at a distance $d . S$ and the circumferential surface $S_{\mathrm{c}}$ are shown separately for clarity. $\mathbf{n}_{\mathbf{S}}$ is the surface normal of $S$, and $\mathbf{n}$ is the surface normal vector of $S_{\mathrm{p}}$ (directed towards $S$ ).

or, the area-averaged sound absorption coefficient. In case of non-plane waves and not purely normal incidence, one obtains an effective sound absorption coefficient.

Numerical investigations [23] showed that, accurate estimates for the normal incidence sound absorption coefficient can be obtained, even in the presence of interfering reflections. Hence, area-averaging seems effective in reducing the effects caused by such reflections. However, it was also observed that the measurement surface preferably needs to lie within $1 \mathrm{~cm}$ from the surface of interest to obtain accurate results, in particular for poorly absorbing surfaces. Measuring at such a small distance is not always possible or practical, considering, for instance, the dimensions of typical electro-acoustic transducers, or nonflatness of the material surface.

In this paper, we investigate the influence of the measurement distance for a poorly absorbing surface on the measured sound absorption coefficient and propose an approach to produce accurate results for practical values of the measurement distance. To first familiarize the reader with the LPW-method, the theory, with formulations for pu- and pp-intensity probes, is presented in section 2 . This section is followed by a theoretical investigation of the sound field in front of an acoustically hard surface in section 3. This investigation shows that measurements at a larger distance are possible if the measurement surface is extended with the circumferential surface surrounding the volume between the material- and the measurement surface. Experimental results that demonstrate the effect of the proposed approach are shown and discussed in section 4. Finally, in section 5, conclusions and recommendations for further work are given.

\section{Theory}

\subsection{Theory of the LPW-method}

In the following, we assume that the sound field satisfies the Helmholtz equation. Furthermore, the $\mathrm{e}^{\mathrm{i} \omega t}$-convention is used, i.e. $p(t)=\operatorname{Re}\left[P(\omega) \mathrm{e}^{\mathrm{i} \omega t}\right]$. Vector quantities are bold-faced, and the explicit dependence of $\omega$ is omitted for quantities in the frequency domain.

The theory is presented based on the configuration shown in Figure 1. We want to obtain an accurate estimate for the area-averaged normal incidence sound absorption coefficient of the surface area $S$, being a part of a general surface $S_{\mathrm{g}}$. To this purpose, we define a measurement surface $S_{\mathrm{p}}$, parallel to $S$ and at a distance $d$ from $S$. Note that the shape of the measurement surface $S_{\mathrm{p}}$ is a good approximation of the shape of $S$ if the distance $d$ is much smaller than the radius of curvature of $S$. As the space between $S$ and $S_{\mathrm{p}}$ is generally source-free, it is reasonable to assume that the normal incidence sound absorption coefficient of $S$ can be accurately estimated if the distance between both surfaces is small and sound incidence is normal or at least near-normal.

For a measurement surface with a certain area, the areaaveraged sound absorption coefficient $\alpha$ is defined as the ratio of active and incident acoustic sound power

$$
\alpha=\frac{W_{\mathrm{ac}}}{W_{\mathrm{in}}},
$$

where $W_{\text {ac }}$ and $W_{\text {in }}$ are the frequency-dependent active and incident acoustic power. The active acoustic power represents the net acoustic power that is transferred through the area towards the physical surface. The incident acoustic power is the active acoustic power that passes through the same area in absence of the physical surface. Formally, $\alpha$ is the effective area-averaged sound absorption coefficient if sound incidence occurs not purely normal over the surface area of interest. Both powers in equation (1) can be calculated by surface integration of their associated intensities over this surface area,

$$
\begin{aligned}
& W_{\mathrm{ac}}=\int_{S_{p}} \mathbf{I}_{\mathrm{ac}} \cdot \mathbf{n} \mathrm{d} S, \\
& W_{\mathrm{in}}=\int_{S_{p}} \mathbf{I}_{\mathrm{in}} \cdot \mathbf{n} \mathrm{d} S,
\end{aligned}
$$

where $\mathbf{I}_{\mathrm{ac}}$ is the active acoustic intensity vector, and $\mathbf{n}$ is the surface normal vector of $S_{\mathrm{p}}$, see figure $1 . \mathbf{I}_{\mathrm{in}}$ is the incident acoustic intensity vector, being the active acoustic intensity vector at the same position, but in a free-field, i.e. without the sample. Measurement of the active acoustic intensity in direction $\mathbf{n}$ can be performed with a pu[24], or a pp-sound intensity probe [25]. However, the incident acoustic intensity cannot be determined directly from a single measurement, because one cannot distinguish between the incident and reflected acoustic field in front of the sample. To be able to estimate the incident acoustic intensity without the need for a separate measurement in a free-field, or without employing an overall sound field model, the acoustic field is locally approximated by employing a local plane wave assumption, as illustrated in Figure 2. In any point on $S_{\mathrm{p}}$, the acoustic field is approximated by an incident plane wave with complex amplitude $A$, traveling in direction $\mathbf{n}$, and a reflected plane wave with complex amplitude $B$, traveling in the opposite direction. Accordingly, the approximated field consists of a spatial distribution of $A$ and one of $B$.

Accordingly, in each point, defined by its spatial coordinate $\mathbf{r}$, upon $S_{\mathrm{p}}$, the complex acoustic pressure $P(\mathbf{r})$ 

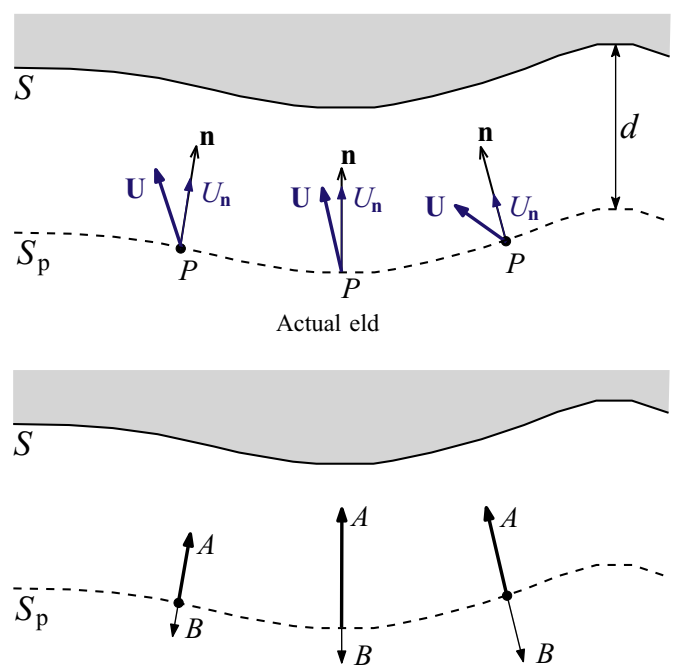

Approximated eld

Figure 2. Upper part: arbitrary acoustic field in front of a material surface $S$; Lower part: approximated field using the local plane wave assumption.

and complex particle velocity in direction $\mathbf{n}, U_{\mathrm{n}}(\mathbf{r})=$ $\mathbf{U}(\mathbf{r}) \cdot \mathbf{n}(\mathbf{r})$, can be written as

$$
\begin{aligned}
P(\mathbf{r}) & =A(\mathbf{r}, \mathbf{n})+B(\mathbf{r}, \mathbf{n}), \\
U_{\mathrm{n}}(\mathbf{r}) & =\frac{1}{\rho_{0} c_{0}}[A(\mathbf{r}, \mathbf{n})-B(\mathbf{r}, \mathbf{n})],
\end{aligned}
$$

where $k=\omega / c_{0}$ is the (real-valued) wavenumber, $c_{0}$ denotes the speed of sound, and $\rho_{0}$ the mass density of the acoustic medium. Solving for $A(\mathbf{r}, \mathbf{n})$ and $B(\mathbf{r}, \mathbf{n})$ yields

$$
\begin{aligned}
& A(\mathbf{r})=\frac{1}{2}\left[P(\mathbf{r})+\rho_{0} c_{0} U_{n}(\mathbf{r})\right], \\
& B(\mathbf{r})=\frac{1}{2}\left[P(\mathbf{r})-\rho_{0} c_{0} U_{n}(\mathbf{r})\right],
\end{aligned}
$$

where the dependency on $\mathbf{n}$ has been removed, as $\mathbf{n}=$ $\mathbf{n}(\mathbf{r})$. From equations (6) and (7) it follows that $A(\mathbf{r})$ and $B(\mathbf{r})$ can be determined by measurement of the acoustic pressure and the component of the particle velocity in direction $\mathbf{n}$. Once $A(\mathbf{r})$ and $B(\mathbf{r})$ are known, the incidentand reflected acoustic intensity in directions $\mathbf{n}$ and $-\mathbf{n}$, respectively, can be calculated by

$$
\begin{aligned}
I_{\text {in }}(\mathbf{r}) & =\frac{|A(\mathbf{r})|^{2}}{2 \rho_{0} c_{0}}, \\
I_{\text {refl }}(\mathbf{r}) & =\frac{|B(\mathbf{r})|^{2}}{2 \rho_{0} c_{0}} .
\end{aligned}
$$

The active power, given by equation (2), is determined by spatial integration of the well-known expression for the active acoustic intensity [25],

$$
I_{\mathrm{ac}}(\mathbf{r})=\frac{1}{2} \operatorname{Re}\left[P(\mathbf{r}) \overline{U_{\mathrm{n}}(\mathbf{r})}\right] .
$$

Summarizing, using the LPW-method, the area-averaged sound absorption coefficient can be determined using a 1D

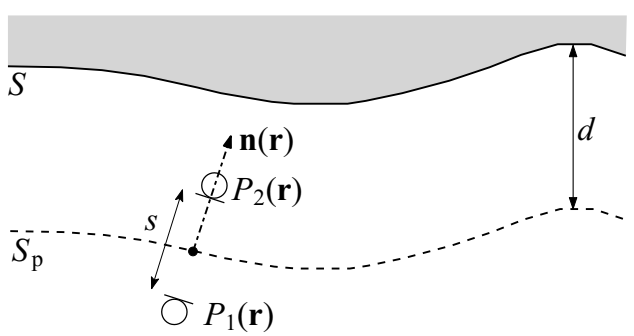

Figure 3. pp-probe with microphone spacing $s$.

sound intensity probe. Accordingly, formulations for puand pp-probes are given in the following two sections, and are presented in terms of power spectral densities, allowing a straightforward implementation on signal processing level.

\subsection{Formulation for a pu-probe}

As the LPW-method is already defined in terms of acoustic pressure and particle velocity, equations (6) and (7) can be substituted directly into equations (8) and (9) to obtain the incident and reflected acoustic intensity. Then, by following the procedure outlined in appendix A1, the spectral density of the incident and reflected acoustic intensity can be expressed as

$$
\begin{aligned}
I_{\text {in }} & =\frac{1}{4}\left[\frac{G_{\mathrm{pp}}}{\rho_{0} c_{0}}+\rho_{0} c_{0} G_{\mathrm{uu}}\right]+\frac{1}{2} \operatorname{Re}\left(G_{\mathrm{pu}}\right), \\
I_{\text {refl }} & =\frac{1}{4}\left[\frac{G_{\mathrm{pp}}}{\rho_{0} c_{0}}+\rho_{0} c_{0} G_{\mathrm{uu}}\right]-\frac{1}{2} \operatorname{Re}\left(G_{\mathrm{pu}}\right),
\end{aligned}
$$

where $G_{\mathrm{pp}}(\mathbf{r}), G_{\mathrm{uu}}(\mathbf{r})$, and $G_{\mathrm{pu}}(\mathbf{r})$ are single-sided autoand cross-power spectral densities of the acoustic pressure and particle velocity. The explicit dependence on the spatial coordinate $\mathbf{r}$ is omitted here for readability. The spectral density of the active acoustic intensity [25] is

$$
I_{\mathrm{ac}}=\operatorname{Re}\left(G_{\mathrm{pu}}\right),
$$

so that the last term in the right-hand side of equations (11) and (12) equals to $\frac{1}{2} I_{\mathrm{ac}}$. One can prove that $I_{\mathrm{ac}}$ can never exceed $I_{\text {in }}$, thus $\alpha \leq 1$. The active and incident acoustic power can now be calculated by spatial integration of both intensities over the projected surface $S_{\mathrm{p}}$, and the area-averaged sound absorption coefficient for is readily obtained by equation (1). One can also use the local active- and incident acoustic intensity to calculate a local sound absorption coefficient, $\alpha_{\text {loc }}(\mathbf{r})$ given by

$$
\begin{aligned}
\alpha_{\mathrm{loc}}(\mathbf{r}) & =1-\frac{|B(\mathbf{r})|^{2}}{|A(\mathbf{r})|^{2}}, \\
& =1-\frac{I_{\mathrm{refl}}(\mathbf{r})}{I_{\mathrm{in}}(\mathbf{r})},
\end{aligned}
$$




\subsection{Formulation for a pp-probe}

Using the setup in Figure 3, a pp-probe can be used to determine the acoustic pressure and particle velocity in the surface normal direction upon the measurement surface $S_{\mathrm{p}}$. Typically, the following equations are used to estimate the complex acoustic pressure $P(\mathbf{r})$ and the complex particle velocity $U_{n}(\mathbf{r})$ at the probe center [25],

$$
\begin{aligned}
P(\mathbf{r}) & \approx \frac{1}{2}\left[P_{1}(\mathbf{r})+P_{2}(\mathbf{r})\right], \\
U_{n}(\mathbf{r}) & \approx \frac{i}{\rho_{0} \omega s}\left[P_{2}(\mathbf{r})-P_{1}(\mathbf{r})\right],
\end{aligned}
$$

where $s$ is the microphone spacing, and $P_{1}(\mathbf{r})=P\left(\mathbf{r}-\frac{s}{2} \mathbf{n}\right)$ and $P_{2}(\mathbf{r})=P\left(\mathbf{r}+\frac{s}{2} \mathbf{n}\right)$, where $\mathbf{r}$ is the spatial coordinate of the probe center. By substitution of equations (15) and (15) in equations (13) and (11), the spectral density of the active and incident acoustic intensity becomes

$$
\begin{aligned}
I_{\mathrm{ac}, \mathrm{FD}}= & \frac{-\operatorname{Im}\left(G_{12}\right)}{\rho_{0} \omega s}, \\
I_{\mathrm{in}, \mathrm{FD}}= & \frac{1}{16 \rho_{0} c_{0}(k s)^{2}}\left\{\left[(k s)^{2}+4\right]\left[G_{11}+G_{22}\right]\right. \\
& \left.+2\left[(k s)^{2}-4\right] \operatorname{Re}\left(G_{12}\right)-8 k s \operatorname{Im}\left(G_{12}\right)\right\},
\end{aligned}
$$

where $G_{11}, G_{22}$, and $G_{12}$ are single-sided power spectral densities of the acoustic pressure signals $p_{1}(t)$ and $p_{2}(t)$, and the subscript ${ }_{\mathrm{FD}}$ indicates that both intensities are calculated using the finite-difference approximation for the particle velocity acc. equation (15).

For sound incidence in the direction of the axis of the pp-probe, one can derive a different formulation. In line with the local plane wave assumption, we can relate the complex acoustic pressures $P_{1}(\mathbf{r})$ and $P_{2}(\mathbf{r})$ to $A(\mathbf{r})$ and $B(\mathbf{r})$ as

$$
\begin{aligned}
& P_{1}(\mathbf{r})=A(\mathbf{r}) \mathrm{e}^{\mathrm{i} k s / 2}+B(\mathbf{r}) \mathrm{e}^{-\mathrm{i} k s / 2}, \\
& P_{2}(\mathbf{r})=A(\mathbf{r}) \mathrm{e}^{-\mathrm{i} k s / 2}+\boldsymbol{B}(\mathbf{r}) \mathrm{e}^{\mathrm{i} k s / 2} .
\end{aligned}
$$

After solving for $A(\mathbf{r})$ and $B(\mathbf{r})$, substitution into equations (8) and (9), application of the procedure in appendix A1, one obtains the spectral densities of the incident and reflected intensity,

$$
\begin{aligned}
I_{\text {in }} & =\frac{G_{11}+G_{22}-2 \operatorname{Re}\left[G_{12} \mathrm{e}^{-\mathrm{i} k s}\right]}{4 \rho_{0} c_{0} \sin ^{2}(k s)}, \\
I_{\text {refl }} & =\frac{G_{11}+G_{22}-2 \operatorname{Re}\left[G_{12} \mathrm{e}^{\mathrm{i} k s}\right]}{4 \rho_{0} c_{0} \sin ^{2}(k s)},
\end{aligned}
$$

where the dependence on $\mathbf{r}$ is again omitted. By evaluating the complex acoustic pressure and particle velocity in terms of $A(\mathbf{r})$ and $B(\mathbf{r})$ at the probe center, and using equation (10), it follows that the spectral density of the active acoustic intensity equals

$$
I_{\mathrm{ac}}=\frac{-\operatorname{Im}\left(G_{12}\right)}{\rho_{0} c_{0} \sin (k s)} .
$$

Equation (23) matches with equation (11) in [26] for the active acoustic intensity in a plane wave sound field in a

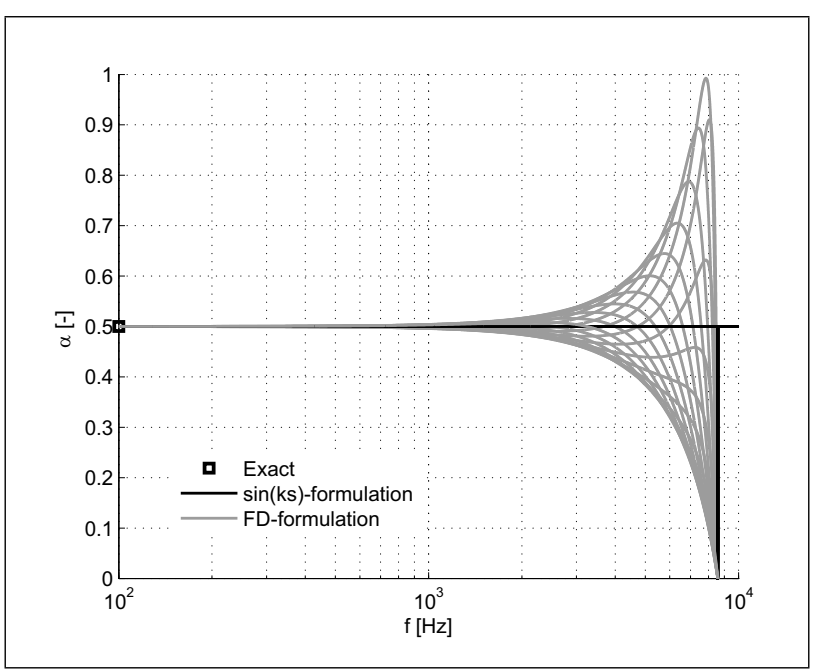

Figure 4. Sound absorption coefficient determined using the FDand the $\sin (k s)$-formulation for normal incidence. Curves are calculated for 20 different phases of the reflected wave where the phase of the incident wave remained constant. Parameters: magnitudes of the complex amplitude of the incident and reflected wave: $1 \mathrm{~Pa}$, and $\frac{1}{2} \sqrt{2} \mathrm{~Pa} . \rho_{0}=1.2 \mathrm{~kg} / \mathrm{m}^{3}, c_{0}=343 \mathrm{~m} / \mathrm{s}$, and $s=20 \mathrm{~mm}$.

duct. For $k s<<1$, the term $\sin (k s)$ may be replaced by $k s$, and, as expected, equation (17) is obtained again.

The effect of using the $\sin (k s)$-formulation in equations (21) and (23) versus the FD-formulation in equations (17) and (19) is clearly visible in Figure 4. In this figure the sound absorption curves are shown for both formulations, for normal plane wave incidence, and a microphone spacing $s=20 \mathrm{~mm}$. The FD-curves rapidly deviate from the exact value $(\alpha=0.5)$ with increasing frequency. Of course, for normal incidence, the $\sin (k s)$-curves are exact, and the usual requirement that the microphone spacing $s$ should be much smaller than the wavelength $\lambda$ can be dropped.

In Figure 4 , at $8.6 \mathrm{kHz}$ a singularity occurs. This singularity is caused by the presence of the $\sin ^{2}(k s)$-term in the denominator of the incident acoustic intensity in equation (21) and occurs if the wavelength equals twice the microphone spacing $s$. One may of course choose a smaller microphone spacing, but the sensitivity to phase-mismatch errors at low frequencies will increase, similar to measurement of the active acoustic intensity with a pp-probe [25]. For the FD-formulation, the absolute error may exceed 0.01 for frequencies above $1500 \mathrm{~Hz}$.

Although the $\sin (k s)$-formulation was derived for incidence in the direction of the pp-probe, similar calculations showed that this formulation leads to an absolute error of less than 0.01 for all frequencies below $5000 \mathrm{~Hz}$ even at an incidence angle of $10^{\circ}$. Considering that the LPW-method is applied for normal or near-normal incidence, we have chosen to use the $\sin (k s)$-formulation and have therefore applied equations (21) and (23) in the preparation of the experimental results shown in section 4 of this paper. 


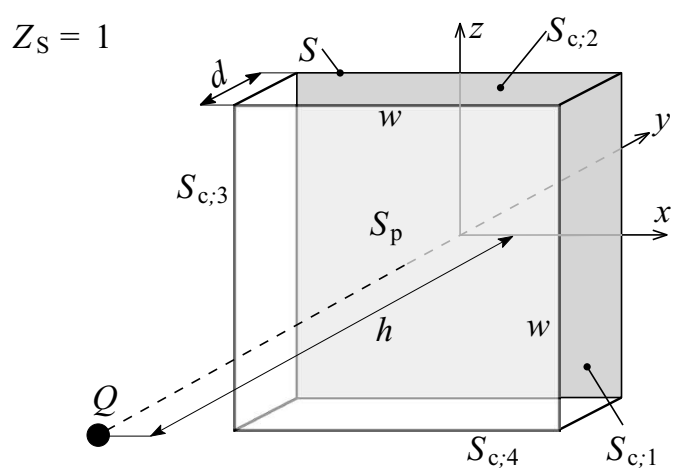

Figure 5. Point source Q located in front of an acoustically hard surface (white). Upon this surface, a material surface area $S$ (dark-gray) is defined; its projected surface at distance $d$ is $S_{\mathrm{p}}$. The $x$ - and $z$-axis both lie upon $S$, whereas the $y$-axis points into the material.

A somewhat more general comparison of the $\sin (k s)$ and FD-formulations for obliquely incident plane waves is given in appendix A2.

\section{Analysis of the sound field in front of an acoustically hard surface}

Previously [23], numerical investigations were performed to investigate the accuracy of the LPW-method. It was found that the accuracy increases with decreasing distance of the measurement surface to the material surface. A high accuracy over the whole simulated frequency range up to $1500 \mathrm{~Hz}$ was reached if the acoustic pressure and particle velocity were both determined within $1 \mathrm{~cm}$ from the material surface.

Furthermore, it was found that the accuracy decreased if the ratio $R_{\mathrm{S}}=A_{\mathrm{p}} / A_{\mathrm{c}}$ of the area $A_{\mathrm{p}}$ of the projected surface to the area $A_{\mathrm{c}}$ of the circumferential surface decreased. This effect was more pronounced in the case the material surface had a high acoustic surface impedance. From these observations, it is expected that the flow of acoustic power through the circumferential area $S_{c}$, see figure 1, can be significant, particularly for poorly absorbing material surfaces. To investigate this hypothesis, we have analyzed the following theoretical example.

We consider a semi-free field bounded by a planar material surface with infinite acoustic surface impedance $Z_{\mathrm{S}}$, subjected to incident sound generated by a point source, see Figure 5. For this case, the (area-averaged) sound absorption coefficient of the material surface area $S$ equals zero per definition. Following a mirror-source approach, the sound field can be calculated analytically. The areaaveraged effective sound absorption coefficient was determined analytically for a square surface area $S_{\mathrm{p}}$ with dimensions $w \times w=256 \times 256 \mathrm{~mm}^{2}$ for different distances $d$ and a source distance $h=1 \mathrm{~m}$. It is shown in Figure 6 for three different values of the distance $d$.

Although one would expect values near zero in Figure 6, values of $\alpha$ up to 0.37 can be obtained at low frequencies

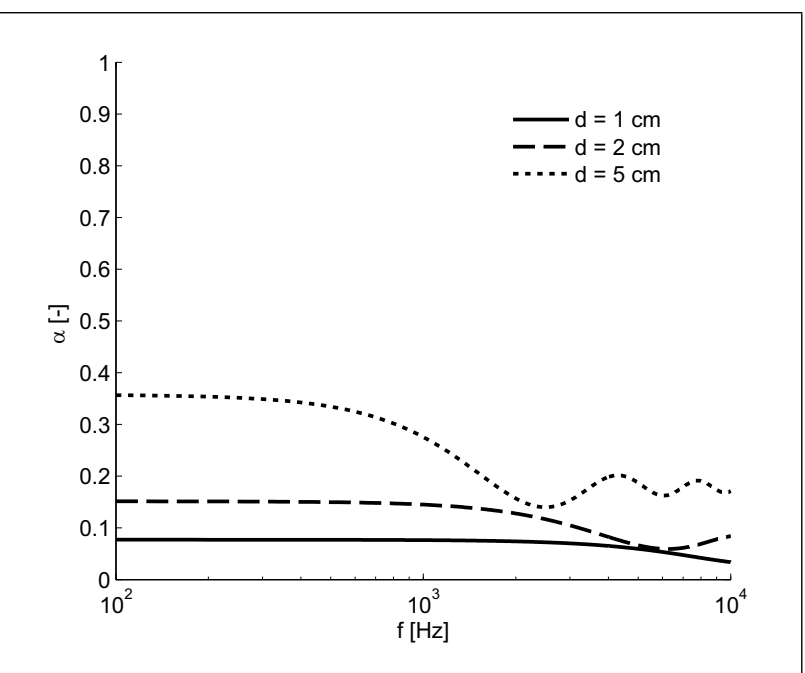

Figure 6. Analytical area-averaged effective sound absorption coefficient of a plane measurement surface, at distance $d$ from a planar acoustically hard surface of infinite extent, irradiated by a point source at a distance of $1 \mathrm{~m}$.

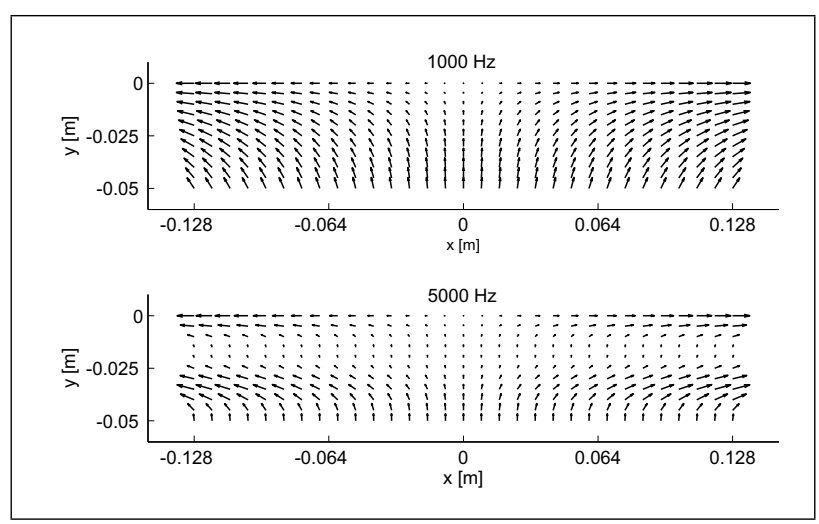

Figure 7. Distribution of the active acoustic intensity over the $x y$-plane within $5 \mathrm{~cm}$ from the material surface. Upper graph: $1000 \mathrm{~Hz}$. Lower graph: $5000 \mathrm{~Hz}$. The material surface is located at the line $y=0 \mathrm{~m}$.

if the distance $d$ is $5 \mathrm{~cm}$. The cause for this behavior lies in the fact that there is a net outflow of acoustic power through the circumferential surface, as illustrated in Figure 7.

To obtain an accurate estimate of the area-averaged sound absorption coefficient of $S$, we have to include the active acoustic power passing through the circumferential surface. I.e., we use the acoustic power balance for the source-free volume between $S_{\mathrm{p}}$ and $S$,

$$
W_{\mathrm{ac}}=W_{\mathrm{ac}, \mathrm{p}}+\sum_{j=1}^{4} W_{\mathrm{ac}, \mathrm{c}, j},
$$

where $W_{\text {ac }}$ is the active acoustic power absorbed by the material surface $S$, and the other powers are defined acc. Figure 8 . If measured accurately, $W_{\text {ac }}$ must equal zero, and thus $\alpha=0$.

As the contribution of the active acoustic power passing through the whole circumferential surface can be ac- 
counted for in this way, the question arises whether this approach should also be followed for the acoustic power that is incident upon this surface. For normal or near-normal incidence, it is expected that the contribution of the power incident upon $S_{\mathrm{c}}, W_{\mathrm{in}, \mathrm{c}}$, will be small compared to the power $W_{\text {in,p }}$ incident upon $S_{\mathrm{p}}$. To verify this, the analytical expressions for both powers were derived,

$$
\begin{aligned}
W_{\mathrm{in}, \mathrm{c}}= & \frac{4|C|^{2}}{\rho_{0} c_{0}}\left\{\operatorname{atan}\left[\frac{2 h}{\sqrt{2 w^{2}+4 h^{2}}}\right]\right. \\
& \left.-\operatorname{atan}\left[\frac{2(h-d)}{\sqrt{2 w^{2}+4(h-d)^{2}}}\right]\right\},
\end{aligned}
$$

$$
W_{\mathrm{in}, \mathrm{p}}=\frac{2|C|^{2}}{\rho_{0} c_{0}} \operatorname{atan}\left[\frac{w^{2}}{2(h-d) \sqrt{2 w^{2}+4(h-d)^{2}}}\right],
$$

where $C$ is the complex amplitude of the acoustic pressure of a point source, as in $P(r, \omega)=\frac{C}{r} \mathrm{e}^{-\mathrm{i} k r}$ with $r=$ $\sqrt{x^{2}+(y+h)^{2}+z^{2}}$. By evaluating these expressions, the ratio $W_{\text {in,c }} / W_{\text {in,p }}$ can be calculated. This ratio is a measure of the relative contribution of power incident upon the circumferential surface $S_{c}$. Figure 9 shows this ratio for two source distances: $h=0.5 \mathrm{~m}$ and $h=1 \mathrm{~m}$. As expected, the relative contribution of $W_{\mathrm{in,c}}$ decreases for an increasing source distance $h$. Furthermore, an approximately linear dependency on $d$ can be observed.

The measurements described in section 4 were all performed with $d=0.02 \mathrm{~m}$ and $h=1 \mathrm{~m}$. According to Figure $9, W_{\text {in,c }}$ then equals ca. $4 \%$ of $W_{\text {in,p. }}$. Correspondingly, $\alpha$ will overestimate the area-averaged effective sound absorption coefficient of $S$ by ca. $4 \%$ if $W_{\text {in,c }}$ is neglected. Although this percentage is significant, for poorly absorbing material surfaces having a sound absorption coefficient below 0.25 , the absolute error will remain below 0.01 . In such cases, where the source distance is sufficiently large, it is justified to ignore the contribution of the acoustic power that is incident upon the circumferential surface.

Therefore, for the measurement of poorly absorbing surfaces using the configuration shown in Figure 5, we propose to determine the active acoustic power upon $S_{\mathrm{p}}$ and $S_{\mathrm{c}}$, and determine the incident acoustic power upon $S_{\mathrm{p}}$ only.

Please note that the considerations in this section are valid for a planar acoustically hard surface of infinite extent. For a panel with finite dimensions, one needs to verify beyond which frequency the mirror source approach leads to a good approximation of the real acoustic field in front of the panel. By using a Fresnel-zone approach, see Heutschi [??], this frequency can be deduced from

$$
d_{c}=\frac{s^{2}}{\pi \lambda}-\frac{\lambda}{4}
$$

where $d_{c}$ is the critical distance, $s$ the panel dimension, and $\lambda$ the wavelength. The critical distance $d_{c}$ is the distance above which the reflection is not purely geometric anymore. Setting $d_{c}=5 \mathrm{~cm}$ and solving for the frequency, it

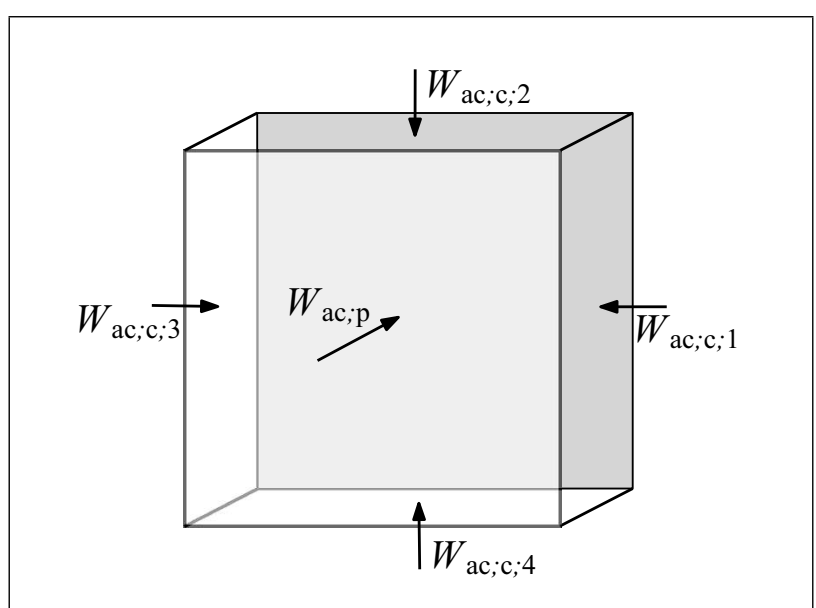

Figure 8. Active acoustic powers of each surface; each power is positive in the directions shown.

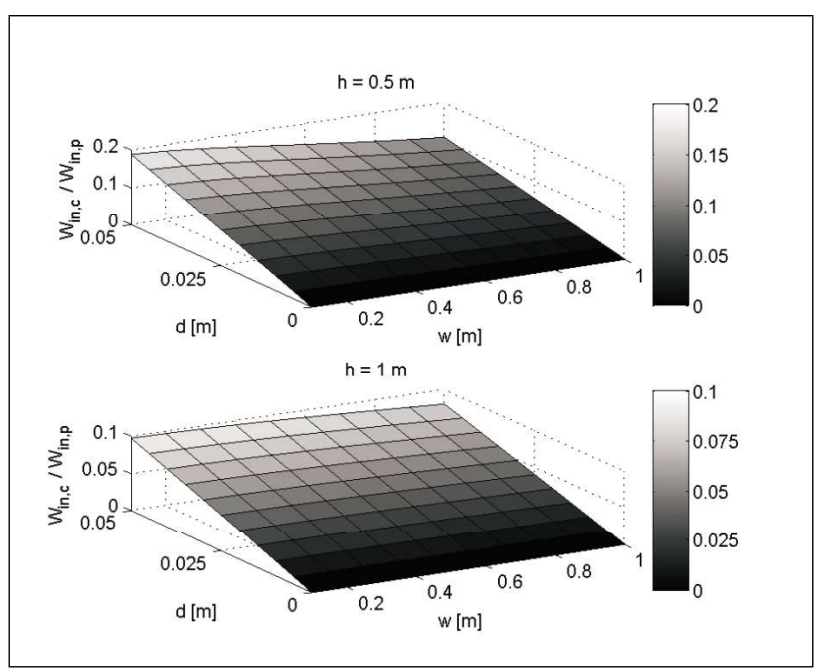

Figure 9. Ratio of $W_{\mathrm{in,c}}$ and $W_{\mathrm{in}, \mathrm{p}}$, as a function of $w$ and the distance $d$.

follows that for normal incidence of plane waves and a distance of $5 \mathrm{~cm}$ from the measurement surface to the physical surface, the critical frequency equals $460 \mathrm{~Hz}$, which is much below the lowest frequency of the experiments discussed in section 4 .

\section{Experiments}

\subsection{Introduction}

In this chapter, the LPW-method is applied to determine the area-averaged normal incidence sound absorption coefficient of a poorly- and a well-absorbing surface. The effect of the proposed extension of the measurement surface upon which the active acoustic power is determined, is investigated. In addition, the influence of the area of the measurement surface is analyzed.

\subsection{Aluminum plate}

The first sample is a $10 \mathrm{~mm}$ thick aluminum plate with dimensions $125 \times 74.5 \mathrm{~cm}^{2}$. The plate was positioned verti- 
cally in a well-absorbing, but not anechoic, room with approximate dimensions $6 \times 6 \times 5 \mathrm{~m}^{3}$. The measurement was performed with a newly developed $3 \mathrm{D}$ pp-probe, consisting of $4 \mathrm{pp}$ sound intensity probes each having 2 MEMS-microphones spaced $20 \mathrm{~mm}$ apart, see Figure 10. These omnidirectional microphones (ADMP441, Analog Devices, $4.7 \times 3.8 \times 1.0 \mathrm{~mm}^{3}$, [??]) offer a low self-noise level, being equal to $33 \mathrm{~dB}(\mathrm{~A})$. This results in a signalto-noise ratio of $61 \mathrm{~dB}(\mathrm{~A})$ at an acoustic stimulus level of $94 \mathrm{~dB}$. Considering the maximum acoustic input level of $120 \mathrm{~dB}$, a dynamic range of $87 \mathrm{~dB}$ results. In practice, the usable dynamic range is somewhat smaller, as this microphone type has a total harmonic distortion of $3 \%$ at a sound pressure level of $104 \mathrm{~dB}$. Data acquisition is performed using a 40-channel front-end and a PC.

The advantage of using very small microphones is that the acoustic center of a single microphone can be placed at only $10 \mathrm{~mm}$ from the plate surface while having sufficient play to avoid any collisions during movement of the probe. Despite the slenderness of the electronic prints and the spacers, calibration measurements showed that some diffraction occurs between 4 and $6 \mathrm{kHz}$. This diffraction is probably caused by the holder upon which the prints are mounted.

The measurement of the active acoustic intensity upon the four parts of the circumferential surface was performed using the four microphones of the probe that are nearest to the plate. As an example, Figure 11 shows how microphones $M_{3}$ and $M_{4}$ are used to determine the active intensity upon $S_{\mathrm{c}, 3}$.

A square measurement grid of $21 \times 21$ points with dimensions $256 \times 256 \mathrm{~mm}^{2}$ was defined upon the plate, resulting in a point spacing of $12.8 \mathrm{~mm}$. An automated scanning system with a positioning accuracy better than $1 \mathrm{~mm}$ was used to move the probe along over the surface of the plate. A small Eurofysica loudspeaker (membrane diameter $23 \mathrm{~mm}$, mounted in a cylindrical housing with a diameter of $80 \mathrm{~mm}$ ) was chosen as a sound source. It was necessary to use such a small speaker in order to resemble a point source so that a comparison of the experimental results with the calculations in section 3 is possible. For the LPW-method, any source can be used. A further advantage of using a small speaker in the experiments is that diffraction by the speaker housing is limited. The speaker was driven with white noise in a wide frequency band, but due to its small dimensions, it hardly radiated sound at frequencies below $1 \mathrm{kHz}$. As a consequence, results are not shown below this frequency.

The source was positioned at $1 \mathrm{~m}$ from the plate, and oriented such that normal incidence occurs at the geometric center of the measurement region. Temperature, ambient pressure and relative humidity were recorded at the start of the scanning session. At each grid point a measurement of $10 \mathrm{~s}$ at a sample rate of $48 \mathrm{kHz}$ was taken. The data was processed in data blocks of 4096 points, with $80 \%$ overlap. The frequency resolution $\Delta f$ of all results equals $11.7 \mathrm{~Hz}$.

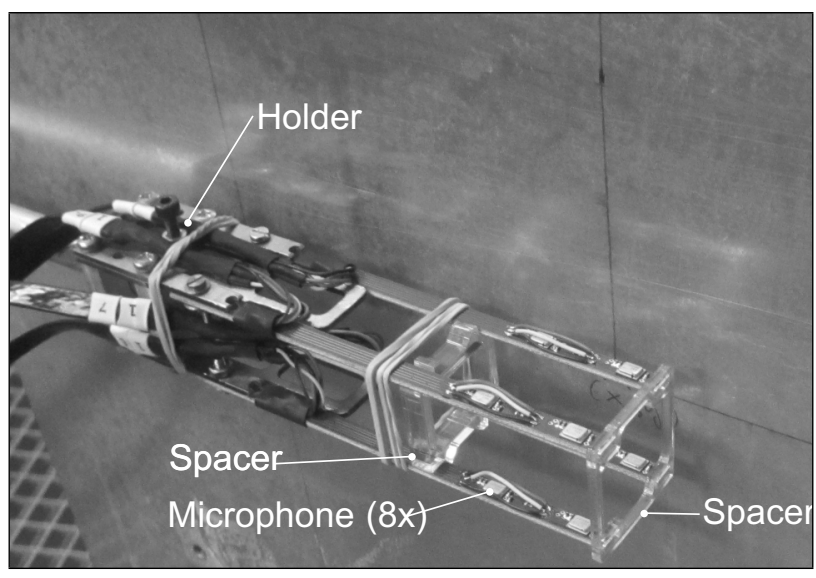

Figure 10. 3D sound intensity probe in front of an aluminum plate.

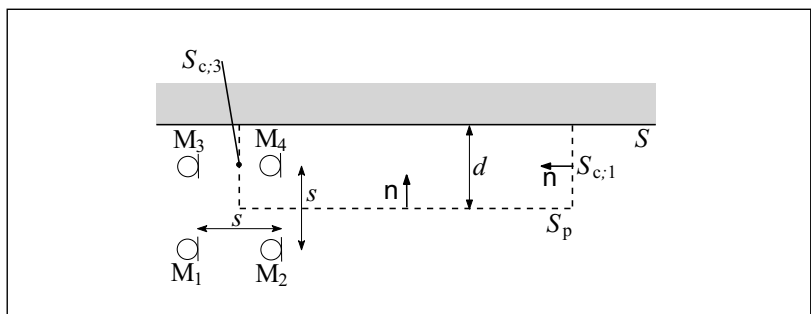

Figure 11. Schematic top view of 3D intensity probe in front of a planar surface.

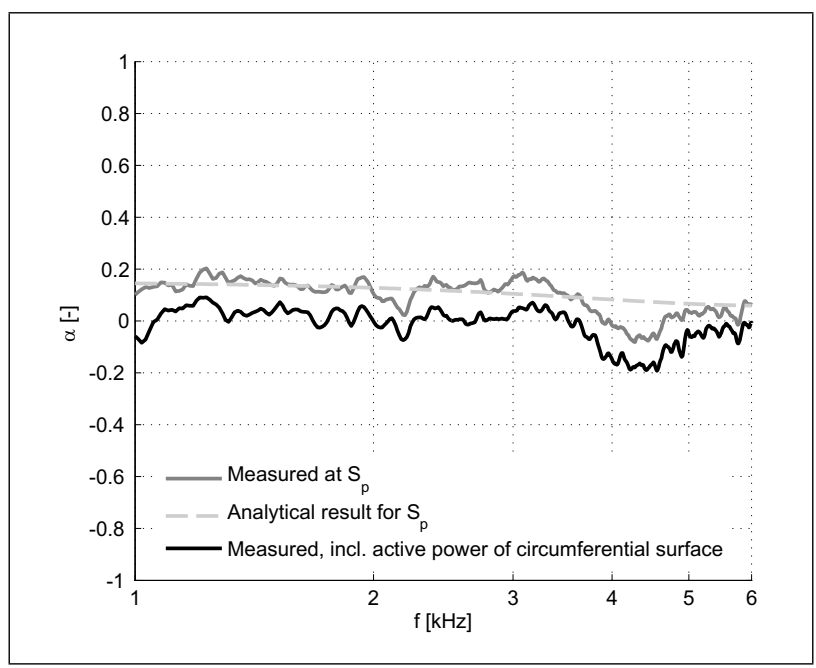

Figure 12. Area-averaged effective sound absorption coefficient of an aluminum plate.

Figure 12 shows the area-averaged sound absorption coefficient, determined using equations (21) and (23). If the flow of active acoustic power through the circumferential area is not accounted for, the solid gray curve is obtained. This curve matches the theoretical, dashed gray, curve for $d=0.02 \mathrm{~m}$ taken from Figure 6 quite well.

The black curve in Figure 12 shows the same result, but now the measurement of the active acoustic power was extended with the circumferential surface. Up to $3.5 \mathrm{kHz}$, this curve represents zero sound absorption on average, as 


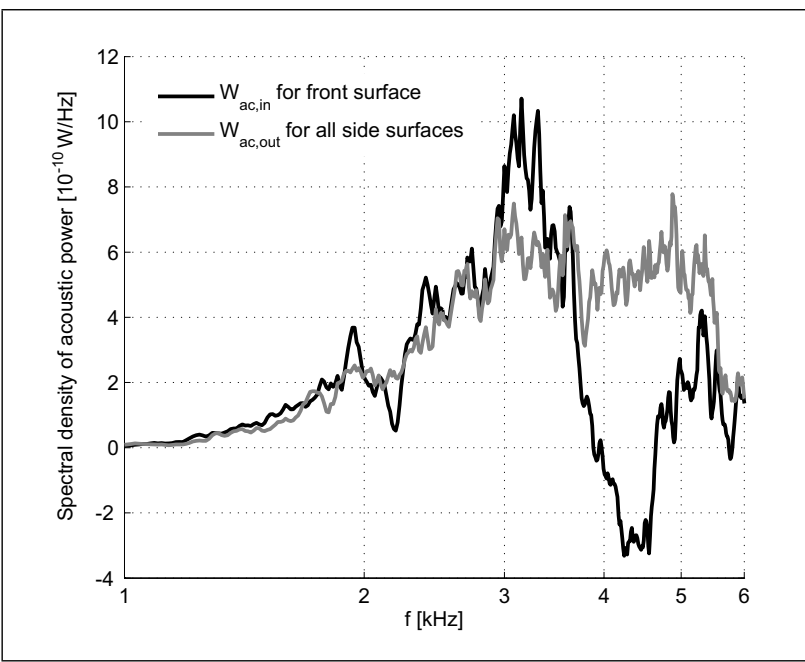

Figure 13. Active acoustic power flowing into $S \mathrm{p}$ and out of the side surfaces.

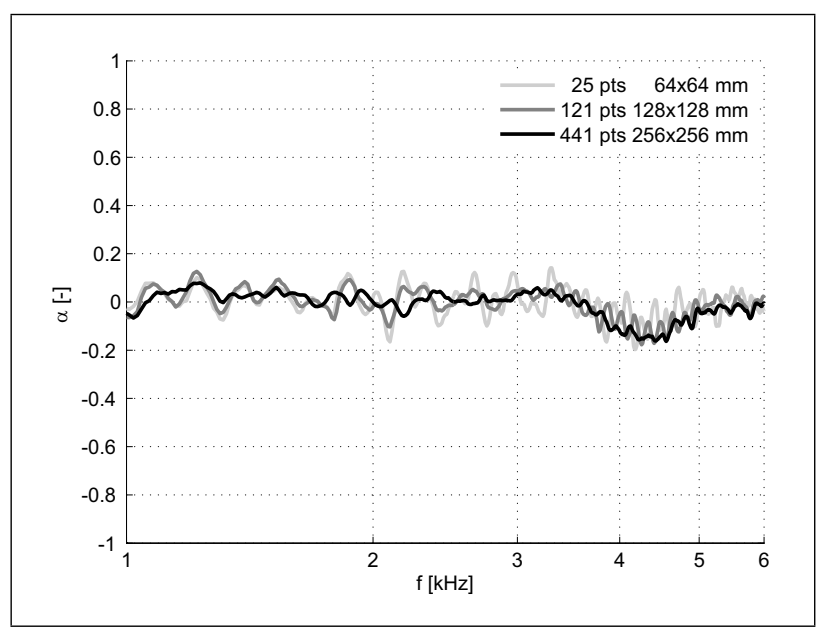

Figure 14. $\alpha$ for three different surface areas.

expected. The variations of both curves may be caused by edge-diffracted waves, acoustic radiation induced by modal response of the plate, or room reflections. Although the room is well-absorbing, such reflections could be induced by large mechanical equipment located in the room. The interferences caused by these phenomena may give rise to negative active acoustic power at some frequencies, therefore negative absorption values are present.

The black curve in Figure 13 shows the active acoustic power flowing into $S_{\mathrm{p}}$. The gray curve represents the active acoustic power flowing out of the circumferential surface, i.e. oppositely to the definition in Figure 8. Theoretically, for an acoustically hard material surface, both curves should coincide. This is approximately the case up to ca. $3 \mathrm{kHz}$, however, above this frequency, the active power flowing into $S_{\mathrm{p}}$ rapidly decreases and even becomes negative. As a result, the sound absorption curves in Figure 12 show a dip between 3.5 and $5 \mathrm{kHz}$. The occurrence of negative active acoustic power in Figure 13, can possibly be explained by amplitude and phase errors caused by the aforementioned diffraction between 4 and $6 \mathrm{kHz}$ of the

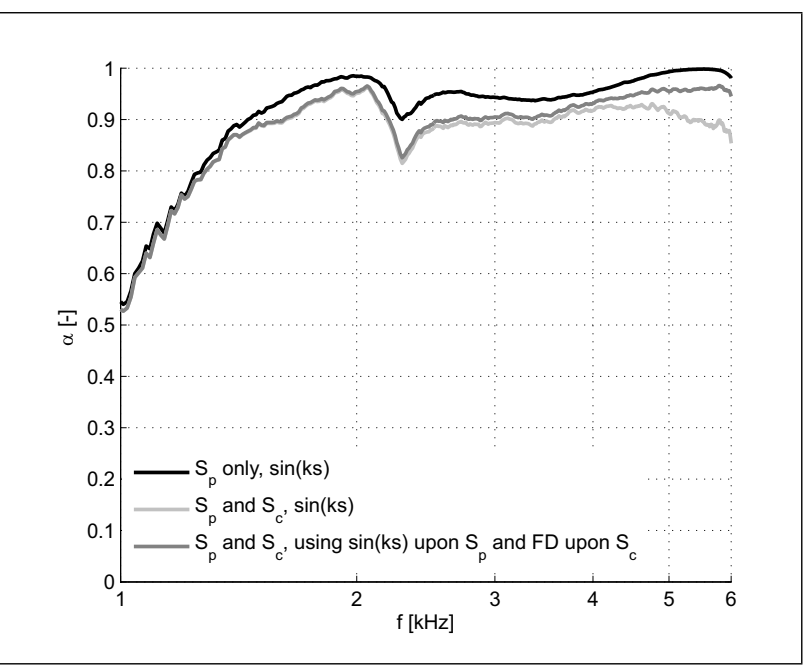

Figure 15. Area-averaged effective sound absorption coefficient of an area of $256 \times 256 \mathrm{~mm}$ of a rigidly backed foam sheet.

probe. Jacobsen [27] found that measurement of the active acoustic intensity in reactive sound fields is very sensitive to such errors.

Although not explicitly noticeable, the gray curve in Figure 13 may as well be influenced by probe diffraction effects. A further possible source of inaccuracy of the active acoustic power flowing through the circumferential surface is the spatial integration of the active acoustic intensity. This integration becomes inaccurate when the variation of the active acoustic intensity with the $y$-coordinate cannot be represented by a linear function anymore between $y=0$ and $y=d$. Looking at Figure 7 , this will already be the case for frequencies starting at a frequency somewhat below $5000 \mathrm{~Hz}$ if $d=20 \mathrm{~mm}$.

The influence of the dimensions of the projected surface was investigated by performing the same analysis as above, but for reduced surface areas, i.e. reduced point sets. Figure 14 shows the area-averaged effective sound absorption coefficient for three different sizes of $S_{\mathrm{p}}$. This graph clearly illustrates that a larger surface leads to less variations in the sound absorption curve. The same behavior was observed in [23]. The explanation for this effect is that interferences (room reflections, diffracted waves) are averaged out more strongly as the surface area increases. All three curves in Figure 14 represent the theoretical value $\alpha=0$ up to $3.5 \mathrm{kHz}$, on average, quite well.

\subsection{Foam sheet}

As a second example, an experiment was performed for a $50 \mathrm{~mm}$ thick sheet of melamine resin foam. Its dimensions are $625 \mathrm{x} 1250 \mathrm{~mm}$, and it was attached to the previously analyzed $10 \mathrm{~mm}$ thick aluminum plate using double-sided adhesive tape. The measurement procedure was identical to the procedure described in the preceding section. Figure 15 shows the area-averaged effective sound absorption coefficient for three cases: 1) only for the projected surface $S_{\mathrm{p}}$ using the $\sin (k s)$-formulation, 2) as proposed in section 3 with the extension of $S_{\mathrm{p}}$ with $S_{\mathrm{c}}$ for the active acoustic 
power using the $\sin (k s)$-formulation for both surfaces, and 3 ) with the extension, but using the FD-formulation for $S_{\mathrm{c}}$ and the $\sin (k s)$-formulation for $S_{\mathrm{p}}$. The latter curve is included as the $\sin (k s)$-formulation becomes inaccurate for combinations of large incidence angles and high frequencies, see appendix A2.

The difference between the black curve for $S_{\mathrm{p}}$ and those for $S_{\mathrm{p}}$ and $S_{\mathrm{c}}$, is smaller than observed for the aluminum plate. This can be explained by the orientation of the active acoustic intensity vector. This vector will mostly be parallel to the circumferential surface for a well-absorbing surface subjected to near-normal incidence, and hence the amount of active power passing through the circumferential surface is relatively less important. Furthermore, it is observed that the FD-formulation indeed leads to a different curve at frequencies exceeding $3 \mathrm{kHz}$.

Figure 16 shows the area-averaged effective sound absorption coefficient (for 3 different surface sizes) and the sound absorption coefficient curve determined with an impedance tube. For the area-averaged measurements, the active acoustic power flowing through the circumferential surface was determined using the FD-formulation. As observed for the aluminum plate, the curves become smoother with increasing surface area. A bit of the variation between the curves can possibly be explained by the variation of the dimensions of the scanned area as the properties of the foam surface vary with position, as will be discussed in the following paragraph. Another explanation could be that the variation of the angle of incidence is larger for a larger measurement surface. However, the maximum angle of incidence equals only $7^{\circ}$ so that the variation of the sound absorption coefficient between $0^{\circ}$ and $7^{\circ}$ is really insignificant if local reaction is assumed for the surface of the foam sheet.

The dips in the curves at approximately $2.27 \mathrm{kHz}$, and a similar one at $6.08 \mathrm{kHz}$ (not shown) are somewhat atypical for the sample under analysis. To investigate their cause, the local absorption coefficient $\alpha_{\text {loc }}$ acc. equation (14) was determined for all grid points for both frequencies and mapped spatially in Figures $17 \mathrm{a}$ and $17 \mathrm{~b}$.

For completeness, the sound absorption coefficient curve obtained with a $50 \mathrm{~mm}$ diameter impedance tube is shown as well. This curve deviates quite much from the other three curves. The largest deviation occurs near the dip in the curves that are determined with the LPWmethod. Nevertheless, a better agreement was expected. A possible reason for the deviations is the omission of the double-sided adhesive layer during the impedance tube test.

Both figures, but particularly Figure $17 \mathrm{~b}$, indicate that absorption locally deviates in vertically oriented zone coinciding with with one strip of adhesive tape that was used to attach the foam. Inspection of the foam after the measurement revealed that it had locally loosened from the adhesive tape. Hence, a small cavity may have been present during the measurements, leading to local change of the sound absorption coefficient. The spatial extent of the absorption deviation is much larger at $2273 \mathrm{~Hz}$ than at

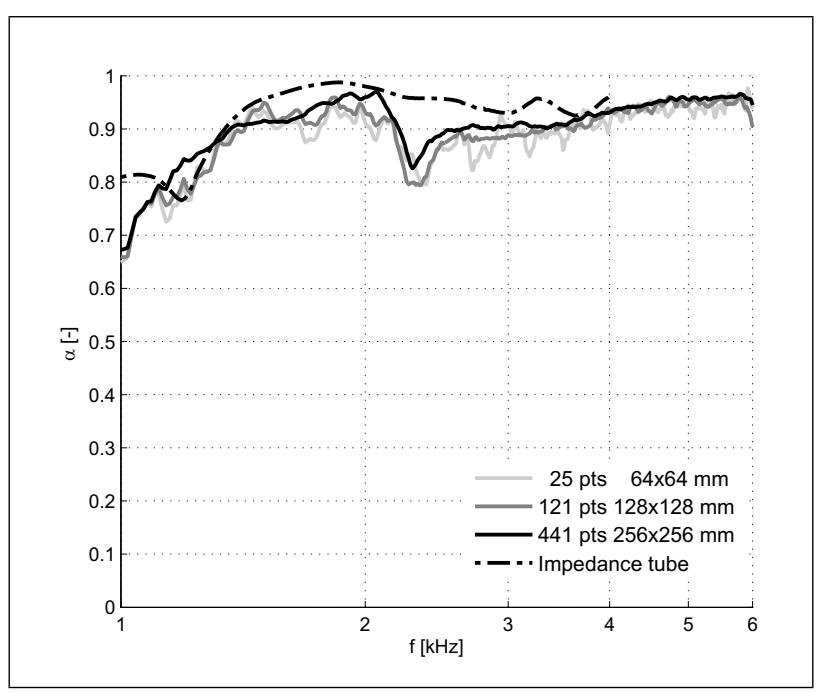

Figure 16. Influence of the surface area.

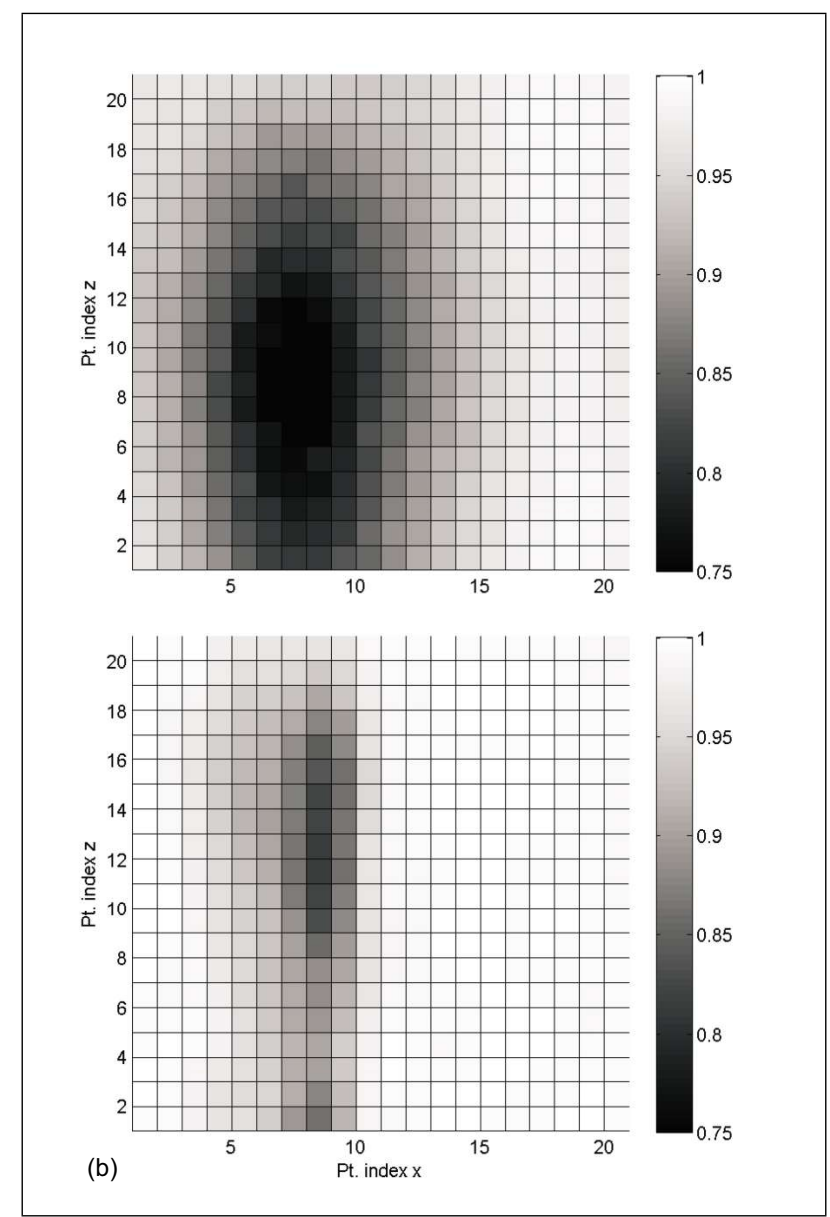

Figure 17. (a) $\alpha_{\mathrm{loc}}$ at $2273 \mathrm{~Hz}$, (b) $\alpha_{\mathrm{loc}}$ at $6082 \mathrm{~Hz}$.

$6082 \mathrm{~Hz}$. This might be related to the acoustical resolution, being dependent on the wavelength. By performing normal incidence sound absorption coefficient measurements for a sample within the affected zone and for a sample outside this zone, in a plane wave tube, it was verified that the variation in the local sound absorption coefficient was 
not caused by a variation of the material properties of the foam itself.

\section{Conclusions and further work}

In this paper, application of the LPW-method for sound absorption measurements is presented. Formulations of the LPW-method for pu- and pp-probes are given. It is shown that the area-averaged effective sound absorption coefficient increasingly rapidly deviates from the theoretical value with increasing distance from the material surface for an acoustically hard surface. It is proposed to compensate for this effect by additionally measuring the active acoustic power that passes through the circumferential surface.

In the determination of the active acoustic power passing through the circumferential surface, the FD-formulation should be preferred over the $\sin (k s)$-formulation as the active acoustic intensity vector generally is not oriented normally or near-normally to the circumferential surface. Furthermore, at the circumferential surface, the measurement grid should be sufficiently dense to avoid spatial integration errors. This requirement theoretically confines the usable frequency range to ca. $4-5 \mathrm{kHz}$ for an acoustically hard surface with the chosen set-up, provided that diffraction of the probe is absent as it may lead to amplitude and phase errors.

Experimental sound absorption measurement of a thick aluminum plate confirms the effectiveness of the proposed approach. The accuracy of the effective area-averaged sound absorption coefficient is increased for this sample. Experimental results for a foam sample showed that the proposed extension is less important for well-absorbing surfaces.

When comparing the LPW-method to existing free-field measurement methods, the most important advantage of the LPW-method is the absence of an overall sound field model. Because the LPW-method does not rely on an overall sound field model of an idealized sound field, it is less sensitive to deviations from the idealized sound field in the real measurement setup. By additionally employing areaaveraging, interference effects that may occur in non-ideal sound fields are reduced. A further argument for using an area-averaged sound absorption coefficient is that the outcome is less prone to spatial variation of the acoustic properties than a point measurement. Therefore, it probably will be a more representative estimate for the surface as a whole. A disadvantage of the proposed method is that the measurement effort is increased compared to existing point-based measurement methods. However, by employing automated scanning equipment or a measurement array, the measurement eff ort can be greatly reduced.

Finally, we recommend to include further investigations with respect to amplitude and phase errors in future work and to conduct in situ measurements to gather experience with the method presented in this paper.

\section{Acknowledgements}

The authors would like to thank CAE Software \& Systems, Gütersloh, Germany, for supplying the prints with the MEMS-microphones. In addition, the support of the Engineering Fluid Dynamics group at the University of Twente during the experiments is gratefully acknowledged.

\section{Appendix}

\section{A1. Formulations in terms of power spectral densi- ties}

The process of switching from a formulation in terms of single-sided spectra to one in terms of single-sided power spectral densities is elucidated in this appendix. We start with equation (10) in which the dependency on the spatial coordinate $\mathbf{r}$ is omitted for briefness,

$$
I_{\mathrm{ac}}=\frac{1}{2} \operatorname{Re}(P \bar{U}) .
$$

This expression is based on the two conventions $p(t)=$ $\operatorname{Re}\left(P \mathrm{e}^{\mathrm{i} \omega t}\right)$ and $u(t)=\operatorname{Re}\left(U \mathrm{e}^{\mathrm{i} \omega t}\right)$. In these conventions, the complex spectra $P, U$, and $I_{\mathrm{ac}}$ are single-sided Fourier transforms. To obtain the single-sided power spectral density (PSD) of the active acoustic intensity, we use the definition for the single-sided cross-power spectral density given by Bendat and Piersol [28, p.55]

$$
G_{x y}(f)=\lim _{T \rightarrow \infty} \frac{2}{T} \mathrm{E}\left[\overline{X_{k}(f, T)} Y_{k}(f, T)\right] .
$$

where $X_{k}(f, T)$ and $Y_{k}(f, T)$ are the double-sided finite Fourier transforms over the $k^{\text {th }}$ record of length $T$. To be able to apply this expression, equation (A1) must first be formulated in terms of double-sided spectra. Following Parseval's Identity, $P=2 P_{\mathrm{ds}}, U=2 U_{\mathrm{ds}}$, and $I_{\mathrm{ac}}=2 I_{\mathrm{ac}, \mathrm{ds}}$ where the subscript ${ }_{d s}$ denotes a double-sided spectrum. equation (A1) then becomes

$$
I_{\mathrm{ac}, \mathrm{ds}}=\operatorname{Re}\left(\overline{P_{\mathrm{ds}}} U_{\mathrm{ds}}\right)
$$

where the conjugation order has been reversed. The power spectral density of $I_{\mathrm{ac}, \mathrm{ds}}(\mathbf{r})$ is now obtained by application of equation (A2),

$$
\begin{aligned}
\lim _{T \rightarrow \infty} \frac{2}{T} \mathrm{E}\left\{I_{\mathrm{ac}, \mathrm{ds}}\right\} & =\lim _{T \rightarrow \infty} \frac{2}{T} \mathrm{E}\left\{\operatorname{Re}\left[\overline{P_{\mathrm{ds}}} U_{\mathrm{ds}}\right]\right\}, \\
& =\operatorname{Re}\left[\lim _{T \rightarrow \infty} \frac{2}{T} \mathrm{E}\left\{\overline{P_{\mathrm{ds}}} U_{\mathrm{ds}}\right\}\right], \\
& =\operatorname{Re}\left[G_{\mathrm{pu}}\right],
\end{aligned}
$$

where the latter expression is generally simply expressed as $I_{\mathrm{ac}}=\operatorname{Re}\left[G_{\mathrm{pu}}\right]$ [25]. Therefore, the relation between the single-sided spectra $P$ and $U$, and the single-sided crosspower spectral density is

$$
\bar{P} U \approx 2 G_{\mathrm{pu}},
$$




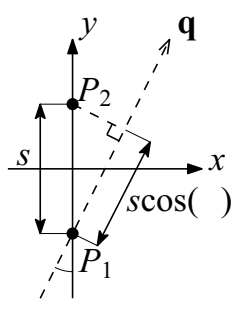

Figure A1. An unidirectional pp-probe (microphones represented by the two black dots) oriented at an angle $\theta$ with respect to the propagation direction $\mathbf{q}$ of a plane wave.

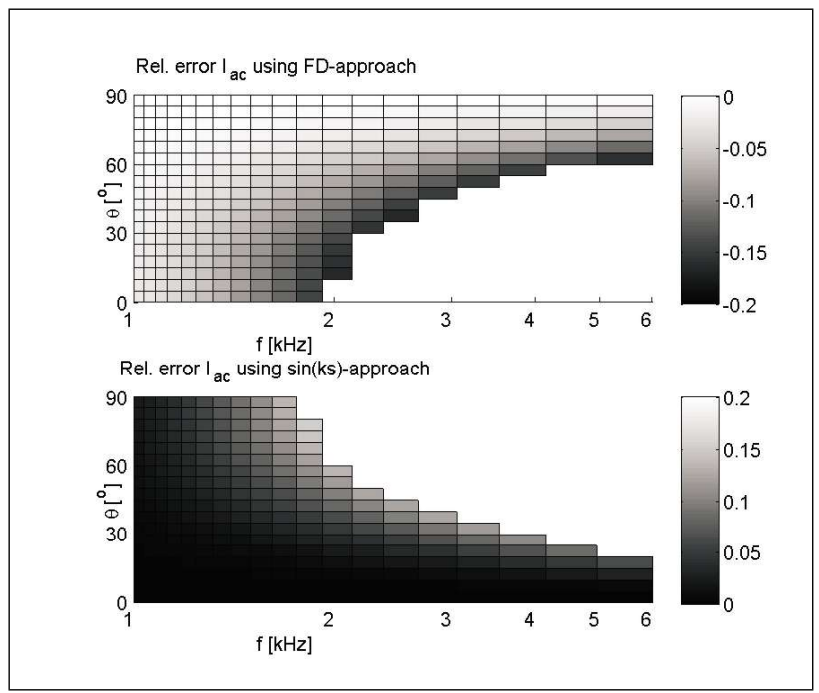

Figure A2. Rel. error $\varepsilon_{\mathrm{FD}}$ and $\varepsilon_{\mathrm{sin}(\mathrm{ks})}$ of the active acoustic intensity vs. frequency and incidence angle for the FD-formulation (upper graph) and the $\sin (k s)$-formulation (lower graph). Data omitted for relative errors greater than \pm 0.2 .

where the approximate equal sign can be replaced with an equal sign, under the assumption that the $G_{\mathrm{pu}}$ is obtained by taking the average of a sufficient number of measurements of an ergodic process. The above procedure may be followed to re-formulate any similar product in terms of single-sided power spectral densities.

\section{A2. Accuracy of measurement of the active acoustic intensity for oblique incidence}

Equation (23) for the active acoustic intensity using the $\sin (k s)$-formulation, is exact for a set of two plane waves, traveling in direction $\mathbf{n}$ and $-\mathbf{n}$. In this appendix, the accuracy of this expression for oblique incidence is analyzed for the case of a single plane wave with complex amplitude $C$, propagating at an angle $\theta$ with respect to the y-axis, see Figure A1. The acoustic pressure and particle velocity can be written as

$$
\begin{aligned}
P & =C \mathrm{e}^{-\mathrm{i} k[x \sin (\theta)+y \cos (\theta)],} \\
U_{y} & =\frac{\cos (\theta)}{Z_{0}} C \mathrm{e}^{-\mathrm{i} k[x \sin (\theta)+y \cos (\theta)]} .
\end{aligned}
$$

The true active acoustic intensity associated with this wave in the y-direction is equal to

$$
I_{\text {ac,true }}=\frac{|C|^{2}}{2 \rho_{0} c_{0}} \cos (\theta) .
$$

Using the $\sin (k s)$-formulation in equation (23), the estimate for the active acoustic intensity becomes:

$$
I_{\mathrm{ac}}=\frac{|C|^{2}}{2 \rho_{0} c_{0}} \frac{\sin [k s \cos (\theta)]}{\sin (k s)} .
$$

Defining the relative error of $I_{\mathrm{ac}}$ as $\varepsilon_{\mathrm{sin}(\mathrm{ks})}=\left(I_{\mathrm{ac}}-\right.$ $\left.I_{\text {ac,true }}\right) / I_{\text {ac,true }}$, one obtains

$$
\varepsilon_{\mathrm{sin}(\mathrm{ks})}=\frac{\sin [k s \cos (\theta)]}{\sin (k s) \cos (\theta)}-1 .
$$

Similarly, for the finite-difference (FD) formulation, using equation (17), one becomes

$$
\varepsilon_{\mathrm{FD}}=\frac{\sin [k s \cos (\theta)]}{k s \cos (\theta)}-1 \text {. }
$$

Figure A2 shows both relative errors as a function of frequency and incidence angle for a pp-probe with a microphone spacing of $20 \mathrm{~mm}$. Whereas the FD-formulation leads to an underestimation, the $\sin (k s)$-formulation overestimates the active acoustic intensity. For a combination of normal incidence and high frequencies, the $\sin (k s)$ formulation is more accurate than the FD-approach. At large angles of incidence and high frequencies the opposite holds.

\section{References}

[1] ISO 354:2003: Acoustics - Measurement of sound absorption in a reverberation room. 2003.

[2] ISO 10534-1: Acoustics - Determination of sound absorption coefficient and impedance in impedance tubes - Part 1: Method using standing wave ratio. 1996.

[3] ISO 10534-2:1998: Acoustics - Determination of sound absorption coefficient and impedance in impedance tubes - Part 2: Transfer-function method. 1998.

[4] K. Attenborough: Propagation of sound above a porous half-space. J. Acoust. Soc. Am. 68 (1980) 1493-1501.

[5] J. Allard, B. Sieben: Measurements of acoustic impedance in a free field with two microphones and a spectrum analyzer. J. Acoust. Soc. Am. 77 (1985) 1617-1618.

[6] Y. Champoux, J. Nicolas, J. Allard: Measurement of acoustic impedance at low frequencies. J. Sound Vib. 125 (1988) 313-323.

[7] Y. Takahashi, T. Otsuru, R. Tomiku: In situ measurements of surface impedance and absorption coefficients of porous materials using two microphones and ambient noise. Appl. Acoust. 66 (2005) 845-865.

[8] N. Din, T. Otsuru, R. Tomiku, N. Okamoto, K. Asniawaty: Measurement method with a pressure-velocity sensor for measuring surface normal impedance of materials using ensemble averaging: Comparison with other methods and its geometrical configuration. Acoust. Sci. Tech. 33 (2012) 86-95. 
[9] M. Garai: Measurement of the sound-absorption coefficient in situ: The reflection method using periodic pseudorandom sequences of maximum length. Appl. Acoust. 39 (1993) 119-139.

[10] E. Mommertz: Angle-dependent in-situ measurements of reflection coefficients using a subtraction technique. Appl. Acoust. 46 (1995) 251-263.

[11] C. Nocke: In-situ acoustic impedance measurement using a free-field transfer function method. Appl. Acoust. 59 (2000) 253-264.

[12] R. Kruse: Application of the two-microphone method for in-situ ground impedance measurements. Acta Acust. Acust. 93 (2007) $837-842$.

[13] R. Lanoye, G. Vermeir, W. Lauriks, R. Kruse, V. Mellert: Measuring the free field acoustic impedance and absorption coefficient of sound absorbing materials with a combined particle velocity-pressure sensor. J. Acoust. Soc. Am. 119 (2006) 2826-2831.

[14] E. Brandão, E. Tijs, A. Lenzi, H.-E. de Bree: A Comparison of three methods to calculate the surface impedance and absorption coefficient from measurements under free field or in situ conditions. Acta Acust. Acust. 97 (2011) 10251033.

[15] E. Tijs, E. Druyvesteyn: An intensity method for measuring absorption properties in situ. Acta Acust. Acust. 98 (2012) 342-353.

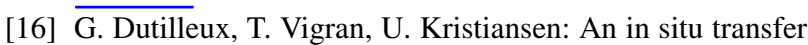
function technique for the assessment of the acoustic absorption of materials in buildings. Appl. Acoust. 62 (2001) $555-572$.

[17] T. Otsuru, R. Tomiku, N. Din, N. Okamoto, M. Murakami: Ensemble averaged surface normal impedance of material using an in-situ technique: preliminary study using boundary element method. J. Acoust. Soc. Am. 125 (2009) 37843791.
[18] K. Hirosawa, K. Takashima, H. Nakagawa, M. Kon, A. Yamamoto, W. Lauriks: Comparison of three measurement techniques for the normal absorption coefficient of sound absorbing materials in the free field. J. Acoust. Soc. Am. 126 (2009) 3020-3027.

[19] P. Dietrich, M. Aretz, M. Müller-Trapet, J. van Gemmeren, M. Vorländer: Uncertainty factors in the determination of acoustic reflection factors with pu-probes. DAGA 2012, Darmstadt, 2012, DEGA, Berlin, 325-326.

[20] Y. Wijnant, E. Kuipers, A. de Boer: Development and application of a new method for the in-situ measurement of sound absorption. ISMA 31, Leuven, Belgium, 2010.

[21] E. Kuipers, Y. Wijnant, A. de Boer: Theory and application of a new method for the in-situ measurement of sound absorption. DAGA 2011, Düsseldorf, 2011, DEGA, Berlin, 723-724.

[22] E. Kuipers, Y. Wijnant, A. de Boer: In situ sound absorption measurement: investigations on oblique incidence. DAGA 2012, Darmstadt, 2012, DEGA, Berlin, 351-352.

[23] E. Kuipers, Y. Wijnant, A. de Boer: A numerical study of a method for measuring the effective in situ sound absorption coefficient. J. Acoust. Soc. Am. 132 (2012) EL236-42.

[24] H.-E. de Bree: An overview of Microflown technologies. Acta Acust. Acust. 89 (2003) 163-172.

[25] F. Fahy: Sound intensity. 2nd ed. E \& FN Spon, London, 1995.

[26] A. Seybert: Two-sensor methods for the measurement of sound intensity and acoustic properties in ducts. J. Acoust. Soc. Am. 83 (1988) 2233-2239.

[27] F. Jacobsen: A note on finite difference estimation of acoustic particle velocity. J. Sound Vib. 256 (2002) $849-859$.

[28] J. Bendat, A. Piersol: Engineering applications of correlation and spectral analysis. J. Wiley and Sons, New York, 1993. 